Note: This is a draft of a paper submitted for publication. Contents of this paper should not be quoted or referred to without permission of the author(s).

Submitted to the Proceedings of the Material Research Society 1999 Fall Meeting, Boston, MA, November 29-December 3. 1999

\title{
Nanocrystal Thickness Information from Z-Stem: 3-D Imaging in One Shot
}

\author{
A. V. Kadavanich, ${ }^{1,2}$ T. Kippeny, ${ }^{2}$ M. Erwin,${ }^{2}$ S. J. Rosenthal, ${ }^{2}$ and S. J. Pennycook \\ ${ }^{1}$ Solid State Division, Oak Ridge National Laboratory, Oak Ridge, TN 37831 \\ ${ }^{2}$ Vanderbilt University, Department of Chemistry, Nashville, TN 37235
}

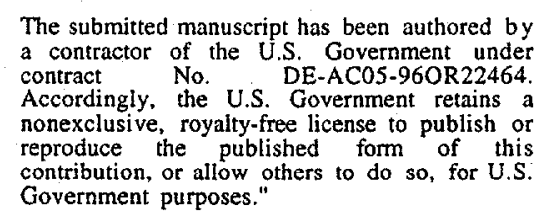

The submitted manuscript has been authored by
a contractor of the U.S. Government under
contract No. NE-AC05-960R22464.
Accordingly, the U.S. Government retains a
nonexclusive, royaity-free license to publish or
reproduce the published form of this
contribution, or allow others to do so, for U.S.
Government purposes."

\author{
prepared by \\ SOLID STATE DIVISION \\ OAK RIDGE NATIONAL LABORATORY \\ Managed by \\ LOCKHEED MARTIN ENERGY RESEARCH CORP. \\ under \\ Contract No. DE-AC05-96OR22464 \\ with the \\ U.S. DEPARTMENT OF ENERGY \\ Oak Ridge, Tennessee
}




\title{
NANOCRYSTAL THICKNESS INFORMATION FROM Z-STEM: 3-D IMAGING IN
} ONE SHOT

\author{
A.V. KADAVANICH ${ }^{*}$, T. KIPPENY*, M. ERWIN*, S. J. ROSENTHAL*, S. J. \\ PENNYCOOK $†$ \\ †Oak Ridge National Laboratory, Solid State Division, Oak Ridge, TN 37831. \\ *Vanderbilt University, Department of Chemistry, Nashville, TN 37235
}

\begin{abstract}
We have applied Atomic Number Contrast Scanning Transmission Electron Microscopy (Z-Contrast STEM) towards the study of colloidal CdSe semiconductor nanocrystals embedded in MEH-PPV polymer films.

For typical nanocrystal thicknesses, the image intensity is a monotonic function of thickness. Hence an atomic column-resolved image provides information both on the lateral shape of the nanocrystal, as well as the relative thickness of the individual columns.

We show that the Z-Contrast image of a single CdSe nanocrystal is consistent with the predicted 3-D model derived from considering HRTEM images of several nanocrystals in different orientations. We further discuss the possibility of measuring absolute thicknesses of atomic columns if the crystal structure is known.
\end{abstract}

\section{INTRODUCTION}

Nanocrystals are an interesting basic research problem and offer many potential applications in optics, electronics and catalysis. The interest is largely due to size-dependent electronic and thermodynamic properties arising from quantum effects or the large surface-tovolume ratio. For instance a $20 \AA$ diameter $C d S$ nanocrystal has band gap that is $50 \%(0.8 \mathrm{eV})$ higher than the bulk crystal due to quantum confinement, ' while the melting point is lowered by several hundred degrees Celsius due to the large surface area ( $\sim 80 \%$ of the atoms are at the surface). ${ }^{2}$

A common problem in the study of nanocrystals is polydispersity. With a few exceptions ${ }^{3,4}$ real nanocrystal samples are characterized by a distribution of sizes and shapes. While modern synthetic techniques have resulted in samples with less than $5 \%$ standard deviation on the average size, ${ }^{5}$ this still implies that bulk characterization techniques provide only an average picture of nanocrystal specimens. Consequently techniques that can characterize individual nanocrystals are of great importance. A variety of electrical and optical measurements ${ }^{6-11}$ have yielded insights into the physical properties of single nanocrystals. Theoretical interpretation of such results requires that the nanocrystal structure be known accurately.

At present, transmission electron microscopy (TEM) is the only technique that can yield atomically resolved structural information. However, due to the difficulty of tilting a nanocrystal specimen through a large angle, such measurements generally only yield a 2-dimensional picture of the nanocrystal structure.3-dimensional models are then built up from analyzing different nanocrystals observed in different orientations. ${ }^{12,13}$ Phase contrast HRTEM in conjunction with image simulations can provide the requisite 3-dimensional information directly if enough data exists to construct a realistic model. However, a more direct technique would be preferred for cases where little a priori information exists.

Z-Contrast STEM is an incoherent imaging process which results in images that are intuitively interpretable in terms of specimen structure and composition. ${ }^{14,15}$ In this paper we demonstrate that for typical nanocrystal samples Z-STEM can provide information on the relative thickness of different parts of a nanocrystal. We also discuss the conditions necessary to achieve absolute thickness determinations and present the results of our initial attempts at accomplishing this.

We focus on CdSe nanocrystals as a test specimen since they are relatively easy to image by Z-STEM and the 3-dimensional structure is reasonably well-established. Furthermore, the binary nature is advantageous for absolute thickness determination, as discussed below.

\section{EXPERIMENT}

Sample Preparation

CdSe nanocrystals were prepared by the method of Murray ${ }^{1.16}$ as modified by Peng ${ }^{5}$ for 
size-focussing. The TOPO surface ligands were exchanged with pyridine by heating in anhydrous pyridine for several hours. The nanocrystals were subsequently precipitated with hexanes and dissolved in chloroform. Poly (2-methoxy,5-(2'-ethyl-hexyloxy)-p-

phenylenevinylene) (MEH-PPV) was prepared by the method of Wudl ${ }^{17}$ and dissolved in chloroform. CdSe samples were stored in a glovebox until use, MEH-PPV was stored under argon in brown glass vials. TEM samples were prepared by mixing the MEH-PPV and CdSe solutions and spin-coating onto single-crystal $\mathrm{NaCl}$ substrates ( $\{100\}$ surfaces). Typical parameters were $20 \mu 1$ of $2 \mathrm{mg}^{\circ} \mathrm{ml}^{-1} \mathrm{MEH}-\mathrm{PPV} / 0.05 \mathrm{mg}^{\circ} \mathrm{ml}^{-1} \mathrm{CdSe}$ solution, spun at $2000 \mathrm{rpm}$. The films were removed by dipping into a water surface, whereupon the film floats onto the surface as the $\mathrm{NaCl}$ dissolves away. The floating films were picked up with lacey carbon coated copper TEM grids (Ted Pella Co.). Film thicknesses were typically in the range from 150-200A as judged from the optical absorption of identical films spun onto glass slides. Specimens for $\mathrm{Z}$-contrast imaging were prepared in air, stored under argon, and loaded in air.

\section{$\underline{\text { STEM }}$}

Z-Contrast imaging was performed in a VG HB603 STEM operating at $300 \mathrm{kV}$ with a nominal resolution of $1.3 \AA$. Bayesian image reconstruction techniques were used to remove noise from the image and deconvolute the resolution function due to the electron beam profile. Most of the analysis is based on a Maximum Entropy (MaxEnt) ${ }^{18-20}$ algorithm running on a dedicated PC with a custom coprocessor card. The details are described elsewhere. ${ }^{14}$ We have also analyzed the data using the Pixon ${ }^{\mathrm{TM}}$ method, a more recent algorithm for image restoration. ${ }^{21,22}$ For presentation, image brightness/contrast was adjusted in NIH Image 1.61, but intensities were measured on the unscaled images.

\section{THEORY}

\section{Z-Contrast STEM}

For normal TEM electron wavelengths, the high-angle scattering is largely incoherent, even for crystalline specimens, ${ }^{15,23.24}$ and becomes more perfectly incoherent as the angle increases. In the limit of perpendicular scattering, coherence is destroyed completely, such that the only restriction on obtaining perfectly incoherent imaging is the ability to detect the scattered radiation. In practical terms, depending on the detector angle, the coherent contribution can vary up to $50 \%$ of the total detected intensity.

For a single atom, pure incoherent scattering is described by the Rutherford scattering formula, which predicts that the beam intensity scattered into a particular angle is proportional to the square of the atomic number $\left(Z^{2}\right)$. This gives Z-STEM its elemental specificity.

It has been shown that for thin crystalline specimens in a channeling orientation, the scattering detected at high angles is predominantly from $1 \mathrm{~s}$ Bloch states of the crystal. These states are highly localized with virtually no transverse coherence. ${ }^{15,23,24}$

Coherence does exist parallel to the beam direction and would result in intensity oscillations as a function of specimen thickness. However, vibrational motion of the atoms destroys the coherence, such that the effect is reduced to a thickness dependent modulation of the incoherent scattering intensity. The intensity of this thermally diffuse scattering is given by Equation 1, reproduced from Pennycook ${ }^{25}$ and the thickness dependence is plotted for the Cd and Se columns of the wurtzite CdSe [100] zone axis orientation in Figure 1.

$$
O_{T D S}(\mathbf{R}, t)=\frac{Z^{2} \varepsilon^{a \nu^{2}}}{2 \mu^{1 s}\left(\xi^{2} \mu^{1 s^{2}}+\pi^{2}\right)}\left[\pi^{2}\left(1-e^{-2 \mu^{1 s} t}\right)-\xi^{2} \mu^{1 s^{2}} e^{-2 \mu^{1 s} t}\left(1-\cos \frac{2 \pi t}{\xi}\right)-\pi \xi \mu^{1 s} e^{-2 \mu^{1 s} t} \sin \frac{2 \pi t}{\xi}\right]
$$

Equation 1: $O_{T D S}=$ Incoherent object function due to the 1s Bloch state as a function of $\mathbf{R}=$ position of an atomic column perpendicular to the beam direction and $t=$ column thickness. $Z=$ atomic number, $\varepsilon^{\text {av }}=$ average excitation of the $1 \mathrm{~s}$ Bloch state, $\mu^{\text {is }}=$ absorption coefficient for 1 s state, $\xi=$ extinction distance.

For CdSe nanocrystals the relevant thickness range is the region below $100 \AA$. As can be seen, the object functions for both $\mathrm{Cd}$ and Se are increasing monotonically in this region. Hence 
the relative intensities of adjacent columns in a Z-STEM image immediately reveal the relative thicknesses of these column. The functions are plotted continuously, but for a crystal they are of course discrete, such that the intensities are quantized.

More importantly, the ratios of $\mathrm{Cd}$ and $\mathrm{Se}$ intensities for columns of equal thickness-as one would expect to find for the dumbbell columns in [100] zone axis,- comprise a discrete, monotonic function of the thickness, and hence can be used to determine absolute thicknesses.

\section{RESULTS/DISCUSSION}

\section{Z-STEM}

The Z-STEM image of a nanocrystal near [100] zone axis orientation is shown in Figure 2. Panel (a) shows the raw image and panel (b) is the MaxEnt reconstruction of the object function with the point spread function of the microscope removed. Panel (c) shows a magnified view of the area in (b) indicated by the square. The dumbbell pairs of $\mathrm{Cd}$ and Se columns spaced $1.5 \AA$ apart are just resolved. The different intensities indicate that the $\mathrm{Cd}$ comprises the top right column of the dumbbell pair. Based on the contrast in the atomic columns the $<001>$ lattice vector direction is assigned as up in the image.

Not all columns are as well resolved within this image. This could indicate strain in the lattice, but it could also be due to image noise. The maximum signal-to-noise ration (SNR) in the raw image is approximately 2 . While the atomic centers are well separated $(1.5 \AA)$ the $1 \mathrm{~s}$ Bloch states have a diameter on the order of $0.8 \AA$ so some overlap between the $S e$ and $C d$ states may be expected. The low SNR then makes it very difficult to accurately resolve the dumbbells. However, all the resolvable dumbbells support the assignment of the $<001>$ direction

It is assumed that the $\mathrm{Cd}$ and Se columns are of equal thickness within each dumbbell column. One may reasonably expect them to differ by one atom at the entrance and exit surfaces each, depending on the exact nature of the surface termination. For the nanocrystal shown the thickness should be on the order of $15-18$ atoms so the maximum error would be $13 \%$ which cannot account for the contrast difference observed. Hence the assignment of the elemental 


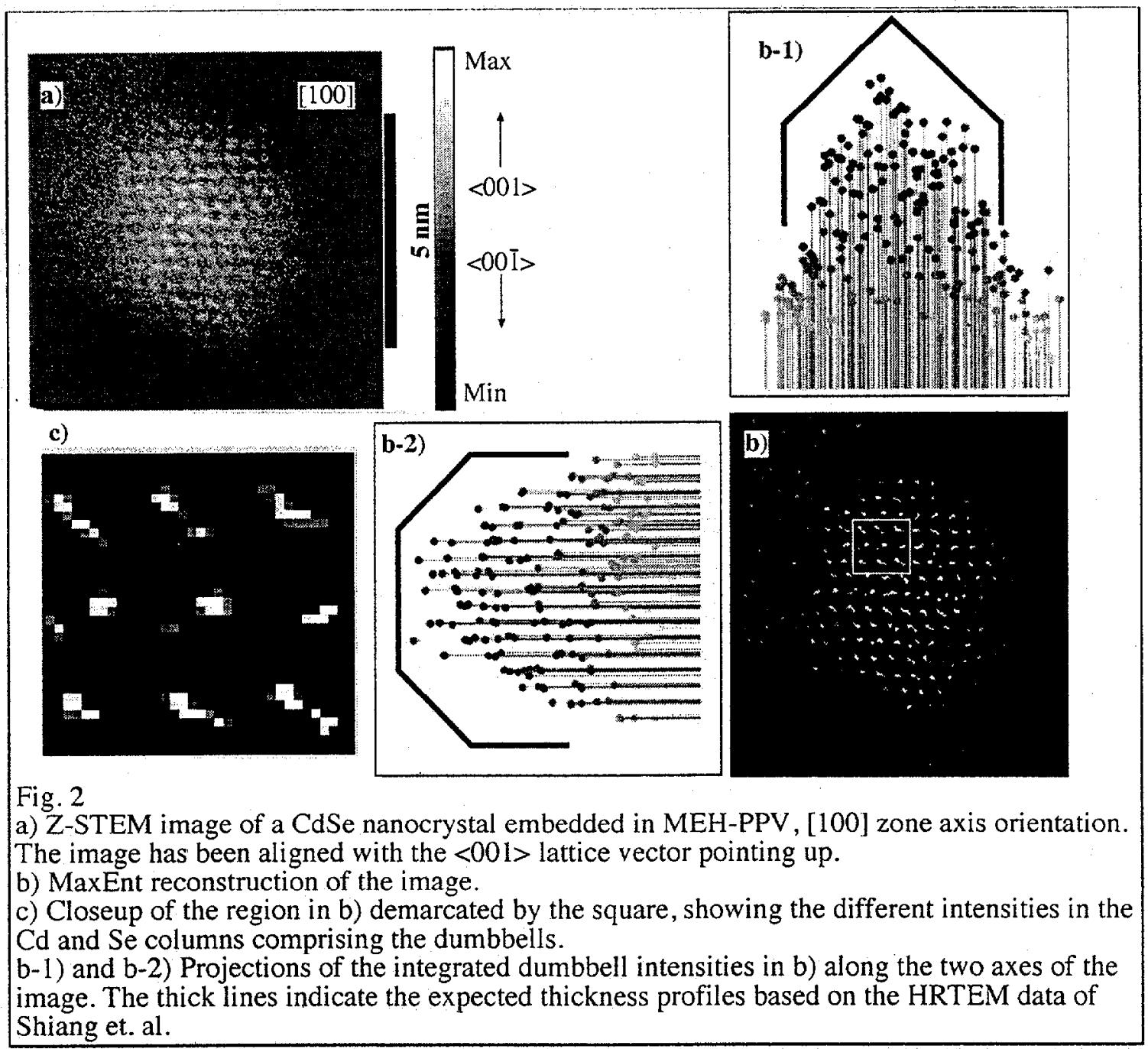

identities is clear-cut.

If we integrate the total intensity of each dumbbell pair we obtain a spatially resolved thickness map, since the dumbbells are compositionally invariant. Projecting the thickness map along the two axes in the image we obtain the thickness envelopes depicted in panels (b-1) and (b-2) of Fig. 2 The thickness envelope corresponds to the expected shape based on previous HRTEM studies on such nanocrystals as indicated in the figure. ${ }^{12}$ However, in this case the three-dimensional information is obtained in concert with the 2-dimensional projection, directly from the image.

We do not observe a discrete quantization of the image intensities as one would expect from Equation 1. This is likely due to the low SNR in the image which makes an accurate reconstruction difficult. Also, as discussed above, if the composition of the dumbbells deviates from 1:1 due to different terminations at the surfaces, the dumbbell intensities will not properly reflect the thickness.

We have calculated intensity ratios for the 14 resolvable dumbbell columns. The range of results varies from 4.1 to 1.0. Based on the intensity ratios in Figure 1 this corresponds to a thickness range of $50 \AA$ to hundreds of $\AA$. While the low SNR results in a fairly large uncertainty in the ratios, estimated at approximately $0.5-1$, this is clearly not reasonable for a nanocrystal whose major diameter in the image plane is $60 \AA$. This discrepancy probably arises from residual coherent scattering reaching the detector. This can be seen from the second set of intensity ratios in Figure 1 which depicts the situation if $20 \%$ of the average intensity reaching the detector is due to coherent scattering. This reduces the lower thickness limit to $20 \AA$, which is a reasonable value for this size of nanocrystal. The contribution of coherent scattering can be suppressed by increasing the HAADF collection angle at the cost of reduced SNR. At present, the overall low 


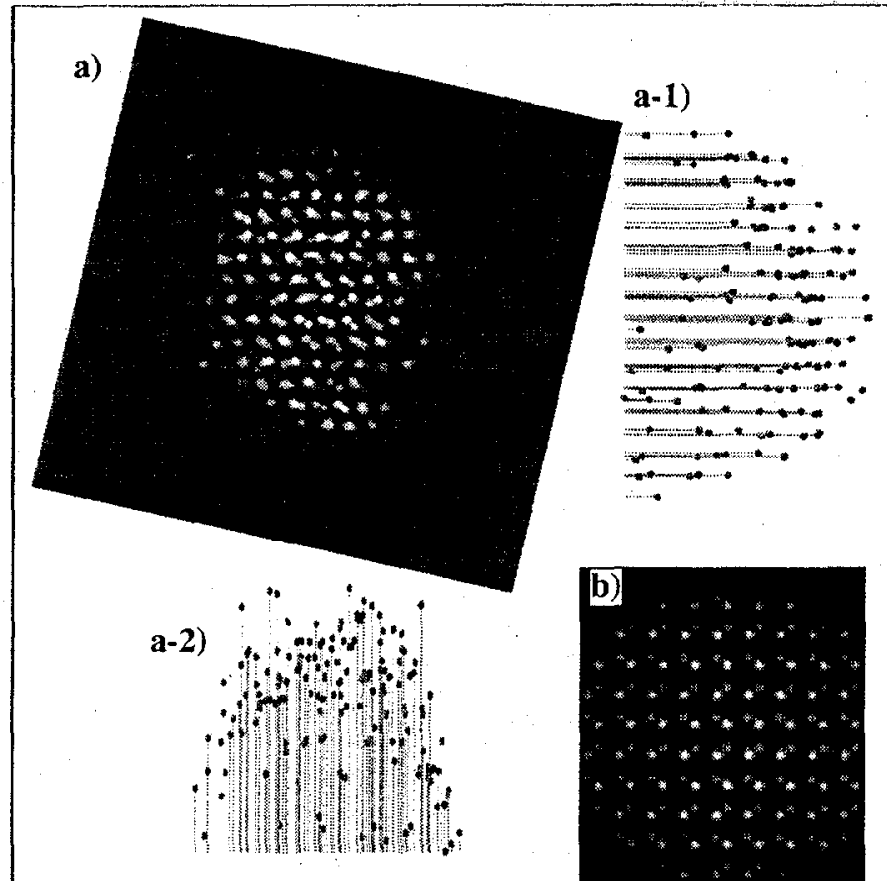

Fig. 3

a) Pixon reconstruction of the nanocrystal shown in Fig. 2

a-1), a-2) Projected intensities of dumbbell columns

from the reconstruction in a)

b) Plot of the 1s Bloch states for the nanocrystal model of Shiang et. al. in [100] orientation.
SNR makes this inadvisable. However, the addition of a spherical aberration corrector to the microscope should provide for adequate SNR, even at large scattering angles. The corrector is scheduled to go online by 2002. In the meantime, improvements in specimen stability may allow us to integrate for longer times and thus keep the SNR within usable limits at higher angles.

The low SNR also makes it difficult to accurately identify the positions of surface atom columns. This is compounded by the inability of the MaxEnt to accurately fit the flat background signal from the polymer, giving rise to spurious column-like features in the region surrounding the particle. The Pixon ${ }^{\mathrm{TM}}$ method, a more recent image reconstruction algorithm ${ }^{21}$ avoids this overfitting resulting in the smoother image shown in Figure 3. In addition to the smoother background, the image more accurately reflects the spatial extent of the Bloch states giving rise to the image. The relative thickness information obtained from this method matches that from the MaxEnt reconstruction of Figure 2 but the intensity ratios are generally lower. While this method seems promising, it is the first time the Pixon ${ }^{\mathrm{TM}}$ method has been applied to Z-STEM data so further testing will be necessary to ensure that the results can stand up to scrutiny.

\section{CONCLUSIONS}

Z-Contrast STEM is capable of resolving the lattice polarity in CdSe nanocrystals and can also provide thickness information directly from the image. By comparing intensities from compositionally similar columns, a map of relative thickness across a nanocrystal is obtained that matches the predicted shape to within the error of the measurement.

Absolute thickness can in principle be obtained from the Z-STEM images. However, this requires data of higher SNR than currently available.

\section{FUTURE WORK}

The Analysis of the Z-STEM data is crucially reliant on obtaining good SNR. In the short term this requires optimizing the specimen preparation to reduce drift and beam damage, allowing longer signal averaging. Presently, beam damage is the limiting factor as the MEH-PPV seems to degrade under the electron beam and cause contamination buildup. We are currently testing an alternate polymer system for use as a matrix.

In the long term the installation of a spherical aberration corrector will result in a beam profile with vastly improved imaging characteristics. The resolution will be improved to $0.5 \AA$ and the SNR is expected to improve by a factor of 7 . The corrector will also reduce tailing in the beam profile, which makes it feasible to attempt column-resolved EELS measurements. The corrector is currently scheduled to be operational in early 2002 and should radically improve the capabilities of Z-STEM microanalysis. 


\section{ACKNOWLEDGEMENTS}

The research presented here was funded by the Department of Energy, Basic Energy Sciences, Materials Sciences Division.

We wish to thank R. Puetter for performing the pixon reconstruction shown. We also wish to thank A. Yahil and Pixon LLC for a research use licence to the commercial Pixon ${ }^{\mathrm{TM}}$ code.

AVK gratefully acknowledges the assistance of P.D. Nellist, B.E. Rafferty and M. F. Chisholm in the operation of the STEM.

\section{REFERENCES}

1. Murray, C.B., Norris, D.J. \& Bawendi, M.G. Journal Of the American Chemical Society 115, 8706-8715 (1993).

2. Goldstein, A.N., Echer, C.M. \& Alivisatos, A.P. Science 256, 1425-7 (1992).

3. Vossmeyer, T., et al. Science 267, 1476-9 (1995).

4. Herron, N., Calabrese, J.C., Farneth, W.E. \& Wang, Y. Science 259, 1426-8 (1993).

5. Peng, X.G., Wickham, J. \& Alivisatos, A.P.Journal Of the American Chemical Society 120, 5343-5344 (1998).

6. Blanton, S.A., Hines, M.A. \& Guyot-Sionnest, P. Applied Physics Letters 69, 3905-7 (1996).

7. Efros, A.L. \& Rosen, M. Physical Review Letters 78, 1110-13 (1997).

8. Empedocles, S. \& Bawendi, M. Accounts of Chemical Research, 389-396 (1999).

9. Klein, D.L., McEuen, P.L., Bowen Katari, J.E., Roth, R. \& Alivisatos, A.P. Applied Physics Letters 68, 2574-6 (1996).

10. Nirmal, M., et al. Nature 383, 802-4 (1996).

11. Tittel, J., et al. Journal of Physical Chemistry B 101, 3013-3016 (1997).

12. Shiang, J.J., Kadavanich, A.V., Grubbs, R.K. \& Alivisatos, A.P. Journal of Physical Chemistry 99, 17417-17422 (1995).

13. Kadavanich, A.V., et al. in Advances in Microcrystalline and Nanocrystalline Semiconductors - 1996 (eds. Collins, R.W.) 353-8 (Mater. Res. Soc, Boston, MA, USA, 1996).

14: Pennycook, S.J., Jesson, D.E., McGibbon, A.J. \& Nellist, P.D. Journal of Electron Microscopy 45, 36-43 (1996).

15. Nellist, P.D. \& Pennycook, S.J. Journal of Microscopy-Oxford, 159-170 (1998).

16. Bowen Katari, J.E., Colvin, V.L. \& Alivisatos, A.P. Journal of Physical Chemistry 98 , 4109-17 (1994).

17. Wudl, F. \& Srdanov, G. in United States Patent (United States of America, 1993).

18. Burch, S.F., Gull, S.F. \& Skilling, J. Computer Vision, Graphics, and Image Processing 23, 113-28 (1983).

19. Skilling, J. \& Bryan, R.K.Monthly Notices of the Royal Astronomical Society 211, 111-24 (1984).

20. Skilling, J. \& Sibisi, S. in Invited and Contributed Papers from the Conference (ed. Johnson, M.W.) 1-21 (IOP, Chilton, UK, 1990).

21. Pina, R.K. \& Puetter, R.C. Publications of the Astronomical Society of the Pacific 105, 630-637 (1993).

22. Puetter, R.C.International Journal of Imaging Systems and Technology 6, 314-331 (1995).

23. Pennycook, S.J. \& Jesson, D.E. Physical Review Letters 64, 938 (1990).

24. Jesson, D.E. \& Pennycook, S.J. Proceedings of the Royal Society of London, Series A (Mathematical and Physical Sciences) 441, 261-81 (1993).

25. Pennycook, S.J. \& Nellis, P.D. in Impact of Electron Scanning Probe Microscopy on Materials Research (eds. Rickerby, D., Valdrè, G. \& Valdrè, U:) (Kluwer Academic Publishers, The Netherlands, 1999). 